\title{
Analysis of ground motion due to moving surface loads induced by high-speed trains
}

\author{
P. Galvín, J. Domínguez* \\ Escuela Superior de Ingenieros, Universidad de Sevilla, Camino de los \\ Descubrimientos s/n, 41092 Sevilla, Spain
}

\begin{abstract}
A three dimensional time domain boundary element approach for the analysis of soil vibrations induced by high-speed moving loads is presented in this paper. An attenuation law is included in the formulation. By doing so, internal material damping can be taken into account. The characteristics of the BE model required for the study of travelling load problems are analyzed. Thus, mesh size, type of elements, internal damping representation, and the complete numerical approach are validated. Existing analytical solutions for some simple problems are used as a reference. Experimental results measured in a simple soil dynamic load problem are also accurately reproduced by the proposed model. The analysis of the type of BE mesh required for a good representation of high-speed train effects is carried out using different discretizations under the sleepers and the free field near the track. All these analyses allow to define a model very well suited for the study of soil vibration effects due to high-speed train passage. Vibrations produced by an Alstom (ThalysAVE) train travelling at $256 \mathrm{~km} / \mathrm{h}$ and $300 \mathrm{~km} / \mathrm{h}$ speed are evaluated at different locations near the track. Results show that the proposed numerical procedure and attenuation law allow for a realistic representation of the effects of the different passing loads. The BE approach presented in this paper can be used for actual analyses of high-speed trains induced vibrations. Layered soils, ballast or coupled vibrations of nearby structure can be included in the model in a straightforward manner.
\end{abstract}

Key words: BEM, Time domain, Transient dynamic, Moving load, Experimental validation

* Corresponding author. Tel.: +34 954487293; fax: +34 954487295.

Email address: jose@us.es (J. Domínguez). 


\section{Introduction}

Engineers interest for soil vibrations due to high-speed moving loads has increased in the last two decades. This interest has been mainly induced by the increasing importance of high-speed trains in modern transportation. New lines have been constructed in many countries in recent years. It is a fact that trains have played an essential role in transportation for more than a century; however, the increase of velocities make necessary to understand wave propagation problems that were not important for traditional trains. Train speed may be close to soil wave propagation velocities; therefore, the analysis and control of waves induced by these high-speed trains is essential nowadays. The study of this soil motion requires comprehensive models that take into account many factors related to local soil properties, topography and discontinuities. Nearby structures should also be included in the model in case soil structure interaction effects are relevant or when vibrations induced on those structures were to be studied. Particular soil conditions, discontinuities, nearby structures and other local factors can only be taken into account using a numerical method.

It is known that Boundary Element Method is well suited for dynamic soilstructure interaction problems since unbounded regions are represented in a natural way [1] and local conditions can be represented without much difficulties. Because of that, a three dimensional time domain formulation of the $\mathrm{BEM}$ is used in the present paper to model the problem of soil motion due to travelling loads. Due to the lack of an explicit half-space fundamental solution for the impulsive force, a full-space fundamental solution is used. This fact makes necessary discretization of the soil surface extended to a limited area around the zone of interest. Nine node quadrilateral and six node triangular quadratic elements are used. Piecewise linear and piecewise constant time interpolation are used for displacements and tractions, respectively. Special attention is paid to stabilizing algorithms and element subdivision to improve efficacy, stability, and accuracy of the procedure. In this paper, a decaying law for wave amplitude is introduced into the BE integral equation in order to represent internal damping of the soil. Details of the general time domain BE approach can be seen in previous work of Marrero and Domínguez [2]

One century ago, Lamb [3] presented for the first time the solution of the problem of waves induced on the soil by a surface time dependent point load. Three types of waves: P-waves, S-waves and surface waves, with wave velocities $c_{p}, c_{s}$ and $c_{R}$, respectively, were identified in this work. Sixty years later, Eason [4] obtained the displacements on the surface of an elastic half-space when a traction applied on a rectangular or circular surface travels at constant speed over the soil surface. The solution was written in terms of integrals that had to be numerically evaluated. Payton [5], and Gakenheimer and Miklowitz [6] 
obtained soil surface and internal displacements, respectively, when a point load is suddenly applied and then travels at constant speed over an elastic half-space surface. Fryba obtained in 1972 the stationary solution for this problem and Barros et al. [8] for a layered half-space. More recently, using Lamb's solution, Barber [9] obtained closed form expressions for normal soil surface displacements due to a point load travelling at constant speed from infinity. Three different situations are distinguished: subsonic, i.e, load speed lower than Rayleigh wave velocity $c_{R}$; transonic, load speed between Rayleigh wave velocity $\left(c_{R}\right)$ and S-wave velocity $\left(c_{s}\right)$ or between S-wave velocity $\left(c_{s}\right)$ and $\mathrm{P}$-wave velocity $\left(c_{p}\right)$; and supersonic, when the load speed is higher than $c_{p}$. The above mentioned analytic or semi-analytic solutions, together with some experimental results obtained by the authors, will be used in this paper to test the proposed approach.

The present paper is intended to set a general and robust technique for transient dynamic problems related to soil surface excitation, that can be used for a variety of problems and in particular for those related to high-speed train passage effects on nearby soil and constructions. To do so, several transient soil surface excitation problems will be analysed. In some cases, results are compared with known analytical solutions. In other cases, values of soil surface motion measured from actual situations are compared with numerical results obtained with the proposed technique. The effects of sleepers, surface topography, underground discontinuities, nearby structures, ballast, etcetera, can be taken into account. The present approach does not require that the problem has constant geometry and soil properties along the travelling load direction as happens in procedures based on a wave number series expansion along this direction (see Madshus and Kaynia [10], Degrande [11] and Jean et al. [12] who use Green's functions presented by Kausel and Roësset [13] or Tadeu and Kausel [14] for 2.5D problems).

In the following, the general formulation of the method for elastic solids is briefly summarized first, then the problem of a travelling load on a elastic half-space is studied. This problem, with known analytical solution, is used to set the numerical parameters and to analyze the kind of mesh required. Second, the technique is extended to soils with internal damping. Numerical results are validates by comparison with results corresponding to a time harmonic surface load on a viscoelastic half-space and with those obtained by the authors by direct measurement of an actual experimental setup. Finally, soil displacement values produced by train travelling at $256 \mathrm{~km} / \mathrm{h}$ and $300 \mathrm{~km} / \mathrm{h}$ are analyzed. All these analyses allow to define a BE model adequate for the study of soil and nearby structures vibrations induced by high-speed trains passage. Some more complex local effects and the influence of the ballast on the motion produced by high-speed train passing will be studied in a forthcoming paper. 


\section{Time domain BEM for 3-D elastic problems}

The three dimensional BE formulation for transient problems is briefly summarized in this section. A complete treatment can be found in the work of Domínguez [1] and Marrero and Domínguez [2].

Starting from the integral representation of displacement $u$ at a point $i$ on the boundary of a elastic body, after space and time interpolation of the boundary variables, piecewise integration in space and time of the fundamental solution kernels and once the boundary conditions are applied, a system of equations can be solved step-by-step to obtain the time variation of the boundary unknowns; i.e. displacements and tractions.

In the present paper, piecewise constant time interpolation functions are used for tractions and piecewise linear functions for displacements, the fundamental solution displacement and traction are evaluated analytically without much difficulty, and nine node rectangular and six node triangular quadratic elements are used for spatial discretization. Each side of the element is divided into equal parts in the natural coordinates domain yielding an element subdivision which is used for numerical integration. The spatial integration extends only to those subdivisions which mid-point is under the effects of the fundamental solution waves according to the causality condition of each term of the fundamental solution. Explicit expressions of the fundamental solution displacements and tractions corresponding to an impulse point load in a 3-D elastic full-space can be seen in the Appendix.

\section{Half-space under constant speed point load}

Motion induced in an homogeneous elastic half-space by a point load travelling at constant speed is studied using the time-domain BE technique summarized in the previous section. To do so, the soil surface is discretized into quadrilateral nine-node elements as shown in Fig. 1. The discretized surface is $86.4 \mathrm{~m}$ long and $2 B=25 \mathrm{~m}$ wide. The load is applied over the $2.5 \mathrm{~m}$ wide mid-band of the discretized surface shown in Fig. 1. The width of the loading band has been chosen equal to the length of a railroad sleeper. Each element in this center band is a $1.2 \mathrm{~m} \times 1.25 \mathrm{~m}$ rectangle. The load is applied prescribing a constant nodal traction value on the five nodes along a line perpendicular to the travelling direction. The soil is assumed to be an elastic half space with the following properties: $c_{p}=238.24 \mathrm{~m} / \mathrm{s}, c_{s}=127.34 \mathrm{~m} / \mathrm{s}$ and $\rho=1850$ $\mathrm{kg} / \mathrm{m}^{3}$. Two different load speed values: $50 \mathrm{~m} / \mathrm{s}$ and $100 \mathrm{~m} / \mathrm{s}$, are assumed. The time step for the boundary element analysis was set $\Delta t=0.012 \mathrm{~s}$ and $\Delta t=0.006 \mathrm{~s}$, for the first and second velocity respectively. By doing so, the 
load line goes from a nodal line to the next each time step. The parameter $\beta=c_{s} \Delta t / L$, where $L$ is the distance between two nodes of an element, takes values $0.34<\beta<2.54$ for the different elements and load velocities.

Vertical displacements at a point on the soil surface located on the vertical symmetry axis perpendicular of the discretization shown in Figure 1 to the load path and at a distance $6.5 \mathrm{~m}$ from the load path axis, are shown versus time in Figures 2 and 3. These figures correspond to load speeds of $50 \mathrm{~m} / \mathrm{s}$ and $100 \mathrm{~m} / \mathrm{s}$, respectively. Both are lower than the Rayleigh-wave velocity $c_{R}=117.94 \mathrm{~m} / \mathrm{s}$ of the soil. The total force applied, resultant from the surface traction is $6 \times 10^{5} \mathrm{~N}$. Numerical results are compared with those computed with the analytical solution presented by Barber [9]. The main differences observed in the figures for the first $0.4 \mathrm{~s}$ are due to the fact that Barber's solution corresponds to a stationary load coming from infinity whereas the present numerical solution corresponds to a load that is suddenly applied at the first time step and then travels from the nodal line at one extreme to the other end of the mesh. Figures 2 and 3 show the perturbation due to this transient effect at the observation point $A$. In fact, regardless of the load speed, the perturbation starts arriving at the observation point at $t=0.18 \mathrm{~s}$ which is the time taken by $\mathrm{P}$-waves to travel from the first load position to the observation point. The maximum perturbation corresponds to the time when S-waves produced by the sudden application of the load arrive at the observation point. It can be seen in Figs. 2 and 3 that except for the initial perturbation, the computed soil displacement matches almost exactly the analytical solution. Taking into account the time symmetry of the problem (as shown by Barber [9]), one can obtain a numerical solution for the surface displacement of the stationary problem by taking the part of the response starting at the moment when the perturbation passes by the observation point (the maximum value) and its symmetric values for times going from 0 to the passing time. By doing so one obtains a very good representation of the surface motion due to a load travelling from infinity at any surface point. Figure 4.a shows the vertical, longitudinal and transversal displacements for the same point at $6.25 \mathrm{~m}$ from the load axis and a load speed of $100 \mathrm{~m} / \mathrm{s}$. Computed vertical displacements are in very good agreement with those obtained from Barber's analytical solution (Figure 4.b).

Surface displacements for travelling load speeds higher than $c_{R}$ can be computed in a similar way. Vertical displacements at the same point $(6.25 \mathrm{~m}$ from the axis) computed using the present $\mathrm{BE}$ approach for a load speed value $v=131 \mathrm{~m} / \mathrm{s}$ such that $c_{s}<v<c_{p}$ are shown in Figure 5 versus the normalized time $\tau=c_{s} t / r$. Displacements are normalized as $G(\tau)=\pi w(t) \mu r / P$, where $w(t)$ represents normal displacements; $\mu$ the shear modulus; $r$ the distance from the observation point to the load axis (equal to $6.25 \mathrm{~m}$ ) and $P$ the load value. Figure 5 shows a good agreement between the computed values and those obtained from Barber's analytical solution. The perturbation due to 
the first application of the load is rather small in this case where the analytical solution has infinite values and a change of sign for the time when Rayleigh waves arrive to the observation point.

Figure 6 shows vertical displacements over an area close to the load when it passes by the center point of the mesh in Fig. 1. Results are shown for four different load velocities corresponding to $0<v<c_{R} ; c_{R}<v<c_{s} ; c_{s}<v<c_{p}$ and $c_{p}<v<\infty$. Soil displacements at each point for $v<c_{R}$, have been plotted from the numerical values computed after the load passes by the point and using the time symmetry of the response for instants previous to the load passing time as done in Figure 4. Thus, the numerical results fully preserve the time symmetry predicted by the analytical solution (Barber [9]), removing spurious perturbations due to transient effects produced by the limited length of the BE mesh. This procedure for spurious noise removal can only be, and has only be, applied for the $v<c_{R}$ case. The symmetry of the soil motion is lost as $v$ increases due to radiation effect of the Mach waves. The typical Mach cone is observed in the case when $v>c_{p}$.

It is worth to point out that the use of a full space fundamental solution with a bounded free surface discretization does not produce significant spurious noise caused by diffraction at the limits of the discretized area. This diffraction effect is really very small as shown by the numerous analyses of dynamic soil structure interaction and soil dynamics problems, carried out using the BEM in the last thirty years.

\section{Internal soil damping}

Up to this point the soil have been assumed to be an uniform purely elastic isotropic half-space. However, it is well known that for actual solids, due to material internal damping, in addition to radiation damping, wave amplitude decrease as waves propagate. Material damping effects in soil motions due to high-speed trains have been experimentally observed by different authors (Madshus and Kaynia [10], Degrande and Schillemans [16], Auersch [17]).

Including viscous material damping in a frequency domain formulation of the BEM is simple [1]. Using a complex valued shear modulus and wave propagation velocities, internal damping is taken into account in a straightforward and easy way. However, considering internal damping in a time domain formulation is not a simple task. Several authors $\underline{[18,19]}$ have proposed different approaches where Laplace or other type of transformation, would allow to introduce internal damping at a very high computational cost. Jin et al. [20] proposed a simple approach for $2 \mathrm{D}$ problems where there is a dominant frequency in the system response. The approach, based on the idea that there is a 
constant amplitude logarithmic decrement per period in a periodic oscillation, is extended here to 3D problems and used for soil response due to high-speed train passage assuming that there is a dominant frequency in the response. The basic idea is rather simple: one only has to introduce a damping term in the boundary integral equation:

$$
\begin{aligned}
c_{l k}^{i} u_{k}^{i}\left(\mathbf{x}^{i}, t\right) & =\int_{0}^{t^{+}}\left[\int_{\Gamma} u_{l k}^{*}\left(\mathbf{x}, t-\tau ; \mathbf{x}^{i}\right) p_{k}(\mathbf{x}, \tau) d \Gamma(\mathbf{x})\right] \exp ^{-2 \pi \xi(t-\tau) / T} d \tau \\
& -\int_{0}^{t^{+}}\left[\int_{\Gamma} p_{l k}^{*}\left(\mathbf{x}, t-\tau ; \mathbf{x}^{i}\right) u_{k}(\mathbf{x}, \tau) d \Gamma(\mathbf{x})\right] \exp ^{-2 \pi \xi(t-\tau) / T} d \tau
\end{aligned}
$$

where the damping term is determined by the viscous damping coefficient $\xi$ or the hysteretic coefficient $\zeta=2 \xi$, and the dominant vibration period $T$ in the response. Once the integral equation is discretized one obtains the usual equation for each time step

$$
\mathbf{H}^{n n} \mathbf{u}^{u}=\mathbf{G}^{n n} \mathbf{p}^{u}+\sum_{m=1}^{n-1}\left(\mathbf{G}^{n m} \mathbf{p}^{m}-\mathbf{H}^{n m} \mathbf{u}^{m}\right) \exp ^{-2 \pi \xi(n-m) \Delta t / T}
$$

where the right hand side term coming from previous steps is damped by an exponential coefficient with linear increasing exponent as time goes on.

Two examples will be used to validate this model, one numerical and one experimental. The first example corresponds to the solution of a space and time harmonic unit amplitude load applied on a band of the soil surface. Figure 7 shows the boundary element discretization where all elements are nine-node quadratic rectangles. This problem was solved by Dasgupta and Chopra [21] with soil properties: $\mu=3.315 \times 10^{6}, \nu=0.33, \rho=2.82 \times 10^{-4}$, in consistent units, hysteretic damping coefficient $\xi=0.25$ and dimensionless frequency $a_{0}=\omega b / c_{s}=0.5$. The obtained normalized vertical displacements are represented versus dimensionless time $t / T$ in Figure 8 for four points at increasing distance from the load. It can be seen from the figure that there is an excellent agreement between the obtained numerical results and the solution presented by Dasgupta and Chopra the only difference being in the transient part of the response at early time steps. It is due to the fact that the present numerical solutions starts from zero initial conditions and the solution in ref. [21] is obtained for a stationary load applied at $t=-\infty$.

The second problem will be used to asses the amount of damping required as compared with an actual situation, and corresponds to the experimental setup shown in Figure 9. A shaker with a loading area $254.8 \mathrm{~mm} \times 320.6 \mathrm{~mm}$ is set on the surface of a sandy soil which can be considered homogeneous. The shaker can oscillate harmonically at different frequencies. Endevco model 86 accelerometers where located on the soil surface at distances $0.5 \mathrm{~m}, 1.0 \mathrm{~m}$, $1.5 \mathrm{~m}$ and $2.0 \mathrm{~m}$ in order to measure vertical displacements. The amplitude of the applied force was obtained from the measured mass and acceleration of 
the shaker. The soil density was $\rho=1850 \mathrm{~kg} / \mathrm{m}^{3}$. The surface wave velocity $c_{R}$ was obtained from passing times of the waves produced by the shaker, at the four accelerometers. The measured value was $c_{R}=166.7 \mathrm{~m} / \mathrm{s}$. Considering this value and a Poisson's ratio $\nu=0.2$, wave velocities $c_{s}=183.47 \mathrm{~m} / \mathrm{s}$ and $c_{p}=299.62 \mathrm{~m} / \mathrm{s}$ were evaluated.

The problem was studied numerically using quadrilateral quadratic boundary elements. A surface of $4.6 \mathrm{~m} \times 5.8 \mathrm{~m}$ was discretized into $36 \times 36$ elements as shown in Figure 10. The load was uniformly applied over the four central elements which have the same surface as the shaker. Numerical values were obtained for different excitation frequencies and assuming several damping ratios. By comparing experimental and numerical results at the four observation points, the soil material damping was determined to be $\zeta=2 \xi=0.1$. Experimental and numerical results are compared in Figures 11 y 12 . They correspond to the vertical displacements measured at distances: $1.0 \mathrm{~m}, 1.5 \mathrm{~m}$ and $2.0 \mathrm{~m}$ from the shaker, respectively. The excitation frequency was $10 \mathrm{~Hz}$ and the amplitude of the applied load $P=402 \mathrm{~N}$. It can be seen from the figures that the numerical results reproduce quite accurately the soil surface response at different points.

Results from the numerical examples presented up to this point show how the proposed time domain BE approach is a very adequate tool to analyze problems of soil vibration due to surface loads. Internal damping is include in a simple way. Even though the last two examples considered correspond to time harmonic excitations with a well defined period, in many other cases a dominant period can be found. In particular, when high-speed induced vibrations are studied, a dominant frequency in the range from $40 \mathrm{~Hz}$ to $80 \mathrm{~Hz}$ is obtained from experimental data. The existence of several frequencies can be sorted out by an average decaying law.

\section{Soil motion due to a high-speed train passage}

The problem of soil vibrations induced by a high-speed train will be studied next. The train is considered to be a set of loads travelling at constant speed. The load is transmitted to the soil by each couple of wheels through the sleepers. A load distribution pattern among several sleepers that transmit the load of an axle, obtained by Krylov [22], is used in this paper. The distribution depends on the rail beam stiffness $E I=12.76 \times 10^{6} \mathrm{Nm}^{2}$ (for two rail beams), the ballast stiffness $k_{s}=250 \mathrm{MPa}$ and the mass per unit length including the sleepers $m=620 \mathrm{~kg} / \mathrm{m}$.

In order to asses the most appropriate BE mesh for the soil surface, a very refined mesh is first assumed for a short length along the track. It is represented 
in Figure 13. It includes the length corresponding to 36 sleepers and a width of $21.28 \mathrm{~m}$, with 960 elements. Each sleeper is discretized into six rectangular elements and so is the surface between two sleepers (Figure 15.a). The rest of the elements are also quadrilateral with increasing size with the increasing distance to the rail beams. The mesh in Figure 13 is very dense and, therefore, able to represent accurately the soil behaviour near the loaded area; but, it is not long enough to represent properly the effect of a load that is travelling from far away and its influence on a given observation point starts much before the load is over this discretized area. It would be necessary to make it several times longer, but this would give an extremely large number of elements. To avoid it, a less dense mesh is tested to represent the soil around the track. The mesh for the same surface is shown in Figure 14. The smallest elements are $1.2 \mathrm{~m} \times 1.25 \mathrm{~m}$ rectangles in a way such that two elements represent the area corresponding to two sleepers and two intermediate areas. A row of 5 nodes is on the mid line of each sleeper (Figure 15.b). In order to compare the two meshes, a load of $15 \times 10^{4} \mathrm{~N}$ travelling at $315 \mathrm{~km} / \mathrm{h}$ from one end of the mesh to the other is assumed. In the first case (Figures 13 and 15.a), the traction corresponding to each sleeper according to Krylov's distribution is applied to the 39 nodes on each sleeper. In the second (Figures 14 and 15.b) this traction is applied to 5 nodes on the same sleepers. It should be taken into account that in this second case, since the shape functions extend over the complete element, the area between two sleepers would also be loaded. An equivalent load is obtained from the relation between the total load under the sleepers according to Krylov's distribution in the first mesh, and the total load that would be transmitted to the soil in the simplified BE model when tractions corresponding to each sleeper in Krylov's model are prescribed at the corresponding nodes. This relation depends on Krylov's distribution which depends itself on the rail beam-ballast-soil properties and the load velocity. In the case considered, with a load speed $315 \mathrm{~km} / \mathrm{h}$, the relation is 0.345 . Taking into account this correction for the second mesh, the vertical and cross displacement components obtained using the two meshes for points of the vertical mid section of Figures 13 and 14, at $2.5 \mathrm{~m}, 4.5 \mathrm{~m}$ and $6.5 \mathrm{~m}$ from the track axis, and a load of $15 \times 10^{4} \mathrm{~N}$ at $315 \mathrm{~km} / \mathrm{h}$, are shown in Figure 16 . The soil is assumed to be a homogeneous elastic half-space with shear modulus $\mu=18.5 \times 10^{6} \mathrm{~N} / \mathrm{m}^{2}$; Poisson's ratio, $\nu=0.3$ and density, $\rho=1850 \mathrm{~kg} / \mathrm{m}^{3}$. It can be seen from the figure that both meshes give almost identical soil surface displacements. This facts lead to the conclusion that elements like those of the coarse mesh can be used to represent the soil surface near the track.

The analysis of the soil vibrations induced by a high speed train is now carried out using the BE mesh of Figure 1 which has a total length of $86.4 \mathrm{~m}$ and elements for the track zone of the same type as those in Figure 14. The train is represented by an array of loads corresponding to the axles location of trains Alstom (Thalys-AVE) shown in Figure 17. Taking into account this load distribution and velocity, displacements at different locations close to the 
track can be computed. The time step for the analysis was set $\Delta t=0.003 \mathrm{~s}$, the total number of steps being 450. The total computer time for the analysis in a laptop computer was $45 \mathrm{~h}$. Displacements of points mid-way along the discretization and distances of $4 \mathrm{~m}, 6 \mathrm{~m}$ and $8 \mathrm{~m}$ from the track axis are represented in Figures 18 and 19 for train speeds of $256 \mathrm{~km} / \mathrm{h}$ and $300 \mathrm{~km} / \mathrm{h}$, respectively. The soil is assumed to have the same elastic properties given above and a viscous damping ratio $\xi=0.04$. The figures clearly show the effect of passing of two axles of the same bogie (two very close peaks), for the point closest to the track, whereas this effect can not be noticed for points at larger distance from the track. The damping of the signal as distance increases and the effect of the larger loads at both ends of the train, are also clearly noticed in the response. The dominant frequency of the soil response near the track obtained from experimental data [16] is in the range from $40 \mathrm{~Hz}$ to 80 $\mathrm{Hz}$ depending on train speed. This value is assumed to be half of the sleeper passing time in the present study $(70 \mathrm{~Hz}$ and $60 \mathrm{~Hz}$ for train speeds of 300 $\mathrm{km} / \mathrm{h}$ and $256 \mathrm{~km} / \mathrm{h}$, respectively).

\section{Conclusions}

The three-dimensional time domain boundary element formulation for elastic solids has been applied to the solution of an important engineering problem: evaluation of soil motion due to high-speed moving loads and in particular, to high-speed trains. To do so it has been necessary to develop some new aspects of the method. First, it has been necessary to include internal material damping in the formulation. A decaying law including a logarithmic decrement for the dominant frequency has been proposed. The damping law has been tested for a simple harmonic loading case and for an experimental setup using the time domain BE formulation. In both cases the behaviour is good. The decaying law has also been used for the study of a high-speed train induced vibrations assuming a dominant period equal to the sleepers passing time.

A type of BE mesh for high-speed train studies has been presented and tested. Results for simple moving loads and high-speed trains at different velocities have been obtained and discussed. All these analyses have allowed to define a BE model well suited for the problem at hand. The present BE approach has been shown to be a very useful tool for high-speed train induced vibrations analysis. More complex situations like: layered soil, effect of ballast, or coupled vibrations of nearby structures will be studied using the present approach in a forthcoming paper. 


\section{Acknowledgements}

The present paper is part of an investigation financed by the Ministerio de Educación y Ciencia of Spain under the research project BIA2004-03955-C0201 and by the Ministerio de Fomento of Spain under the research project "Evaluación de efectos dinámicos del ferrocarril". The financial support is gratefully acknowledged.

\section{References}

[1] Domínguez J. Boundary elements in dynamics. Southampton, Boston, London, New York: Computational Mechanics Publications and Elsevier Applied Science; 1993.

[2] Marrero M. Domínguez J. Numerical behavior of time domain BEM for three-dimensional transient elastodynamic problems. Engineering analysis with boundary elements 2002; 27: 39-48.

[3] Lamb H. On the propagation of tremors over the surface of an elastic solid. Phil. Trans. Roy. Soc. London, Ser. A, 1904; CCIII 1: 1-42.

[4] Eason G. The stresses produced in a semi-infinite solid by a moving surface force. Int. J. Eng. Sci. 1965; 2: 581-609.

[5] Payton RG. An application of the dynamic Betti-Rayleigh reciprocal theorem to moving point loads in elastic media. Quarterly of Applied Mathematics 1964; 21: 299-313.

[6] Gakenheimer DC. Miklowitz J. Transient excitation of an elastic half space by a point load travelling on the surface. Journal of Applied Mechanics ASME 1969; 36: $505-515$.

[7] Fryba L. Vibration of solids and structures under moving loads. Groningen, The Netherlands: Noordhoff International Publishing; 1972.

[8] de Barros FCP. Luco JE. Response of a layered viscoelastic half-space to a moving point load. Wave motion 1994; 19: 189-210.

[9] Barber JR. Surface displacements due to a steadily moving point force. Journal of Applied Mechanics ASME 1996; 63: 245-251.

[10] Madshus C. Kaynia AM. High-Speed Railway Lines On Soft Ground: Dynamic Behaviour At Critical Train Speed. Journal of Sound and Vibration 2000; 231: 689-701.

[11] Degrande G. Free field vibrations during the passage of a high speed train: experimental results and numerical predictions. In: Krylov V, editor. Noise and vibration from high-speed trains. London: Thomas Telford Publishing, 2001. 
[12] Jean P. Guigou C. Villot M. A 2.5D BEM Model for Ground-Structure Interaction. Building Acoustics 2004; 11: 157-173.

[13] Kausel E. Rosset JM. Stiffness matrices for layered soils. Bulletin of the Seismological Society of America 1981; 71: 1743-1761.

[14] Tadeu A. Kausel E. Green's function for two-and-a-half-dimensional elastodynamic problems. Journal of Engineering Mechanics 2000; 126: 10931097.

[15] Mantic V. A new formula for C-matrix in Somigliana identity. Journal of Elasticity 1993; 33: 191-201.

[16] Degrande G. Schillemans L. Free field vibrations during the passage of a Thalys HST at variable speed. Journal of Sound and Vibration 2001; 247: 131-144.

[17] Auersch L. The excitation of ground vibration by rail traffic: theory of vehicletrack-soil interaction and measurements of high-speed lines. Journal of Sound and Vibration 2004; 284: 103-132.

[18] Wolf JP. Darbre GR. Time-domain boundary element method in viscoelasticity with application to a spherical cavity. Soil Dynamics and Earthquake Engineering 1986; 5: 138-148.

[19] Gaul L. Schanz M. Dynamics of viscoelastic solids by boundary element approaches in time domain. European Journal of Mechanics A/Solids 1994; 13: $43-59$.

[20] Jin F. Pekau OA. Zhang CH. A 2-D time domain boundary element method with damping. International Journal for Numerical Methods in Engineering 2001; 51: 647-661.

[21] Dasgupta G. Chopra AK. Dynamic stiffness matrices for viscoelastic half space. Journal of Engineering Mechanics Division ASCE 1979; 105: 729-745.

[22] Krylov V. Effects of track properties on ground vibrations generated by highspeed trains. Acustica-Acta Acustica 1998; 84: 78-90.

[23] Eringen AC. Suhubi ES. Elastodynamics, vol. II. New York: Academic Press; 1975. 


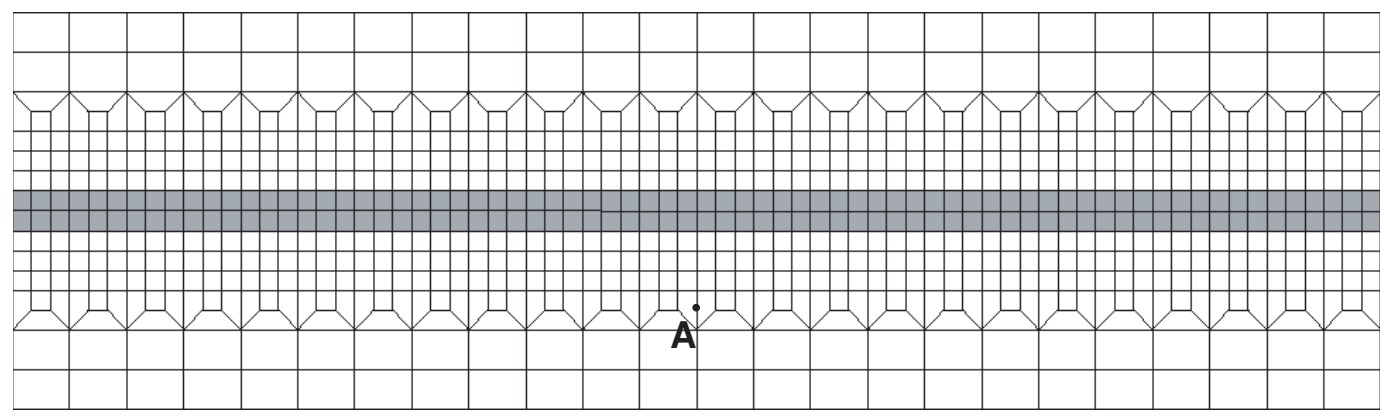

Fig. 1. Soil surface discretization. A: observation point. 


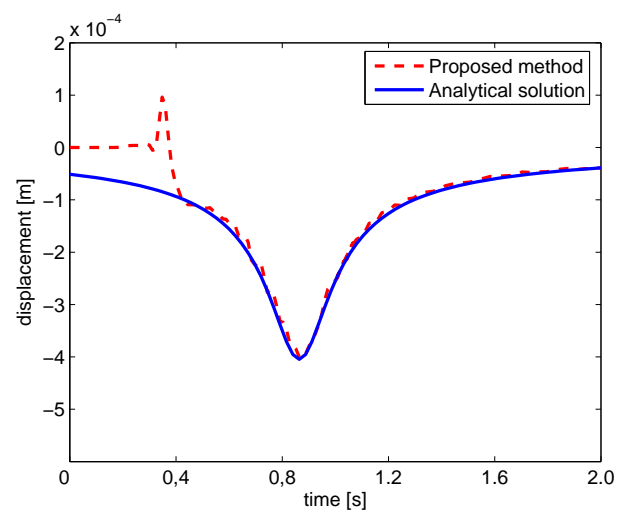

Fig. 2. Vertical displacement at $6.25 \mathrm{~m}$ from the load axis. $v=50 \mathrm{~m} / \mathrm{s}$. 


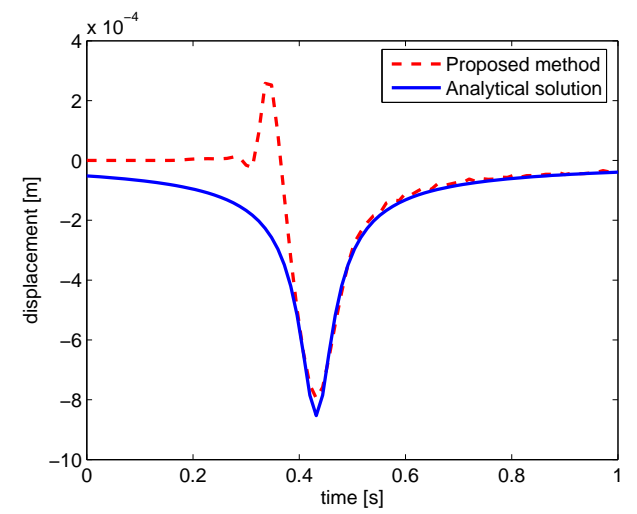

Fig. 3. Vertical displacement at $6.25 \mathrm{~m}$ from the load axis. $v=100 \mathrm{~m} / \mathrm{s}$. 


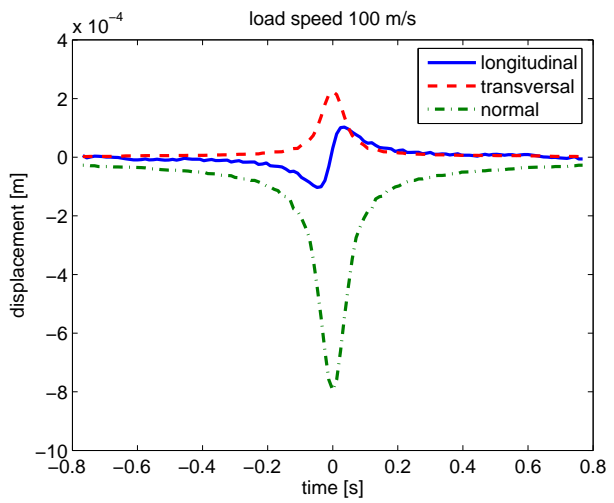

(a) Displacement components at $6.25 \mathrm{~m}$ from the load axis.

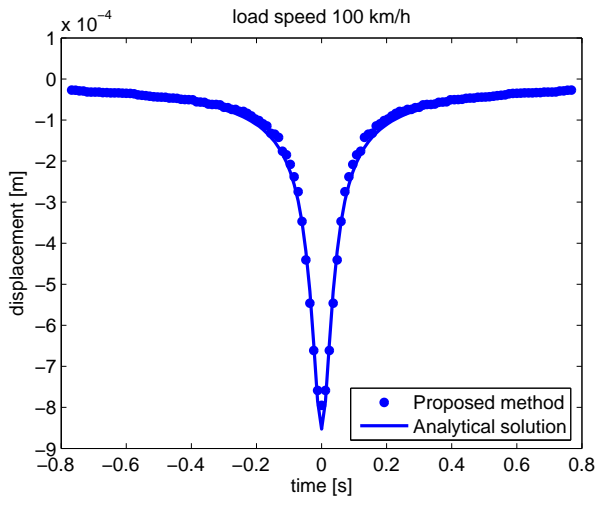

(b) Vertical displacement.

Fig. 4. 


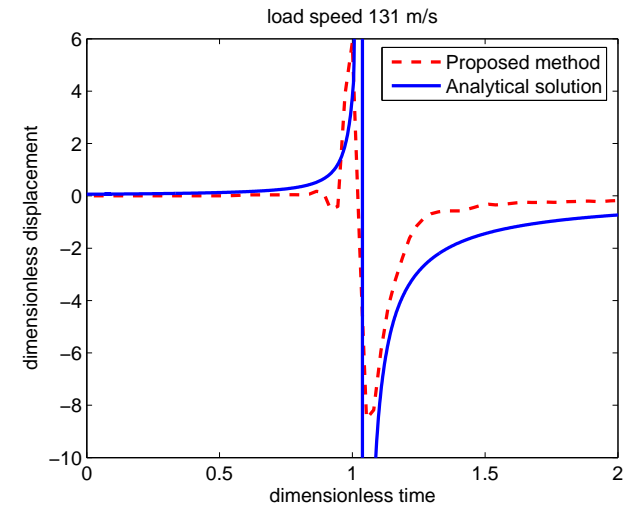

Fig. 5. Vertical displacement at $6.25 \mathrm{~m}$ from the load axis. $v=131 \mathrm{~m} / \mathrm{s}$. 


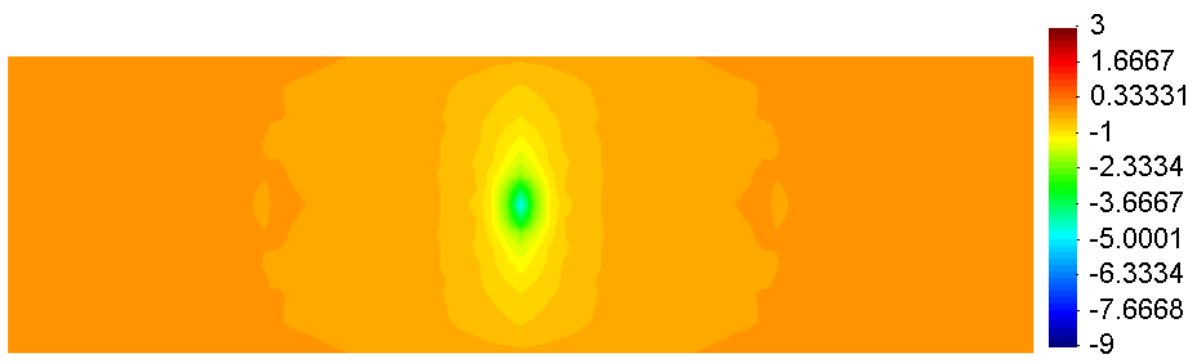

(a) $0<v<c_{R}$.

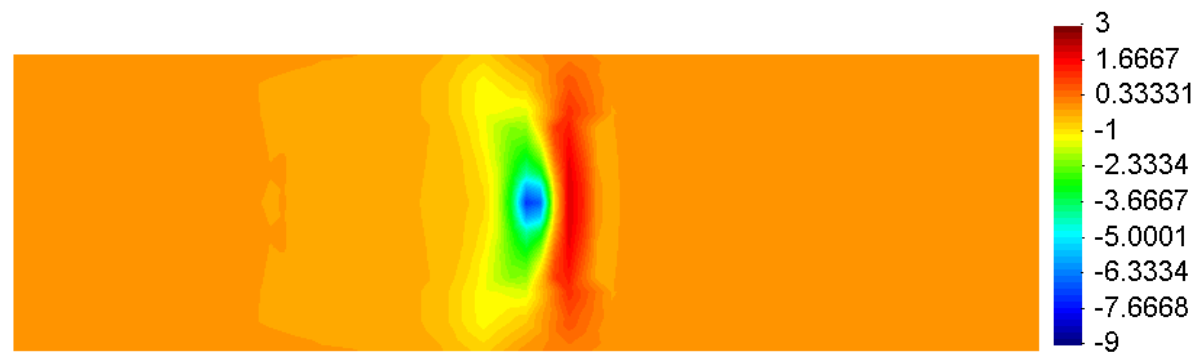

(c) $c_{R}<v<c_{s}$.

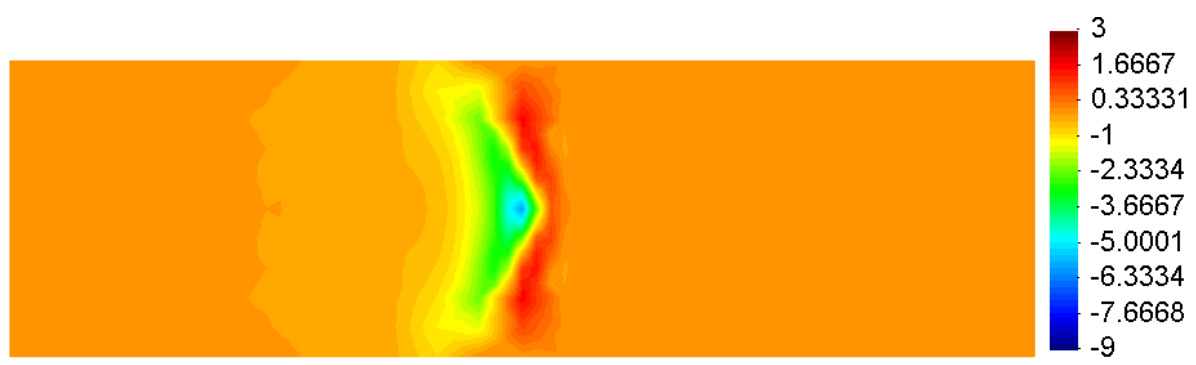

(e) $c_{s}<v<c_{p}$.

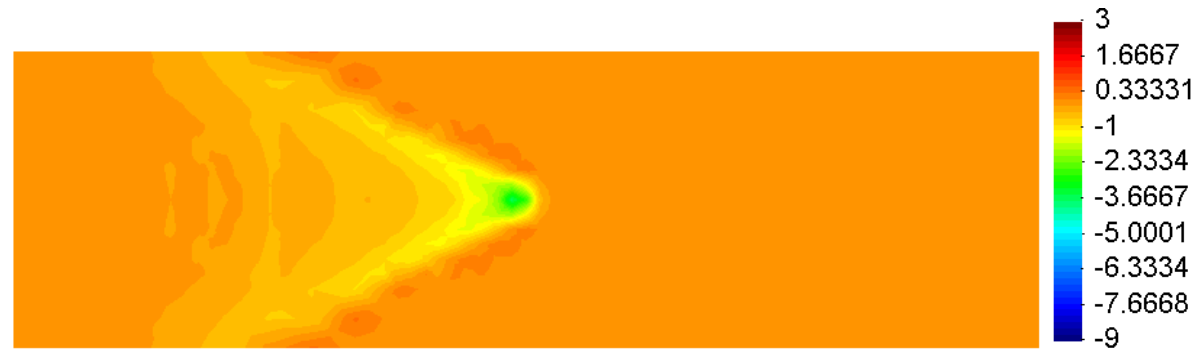

(g) $c_{p}<v<\infty$.

Fig. 6. Vertical soil surface dimensionless displacements $G(\tau)=\pi w(t) \mu B / P$. 


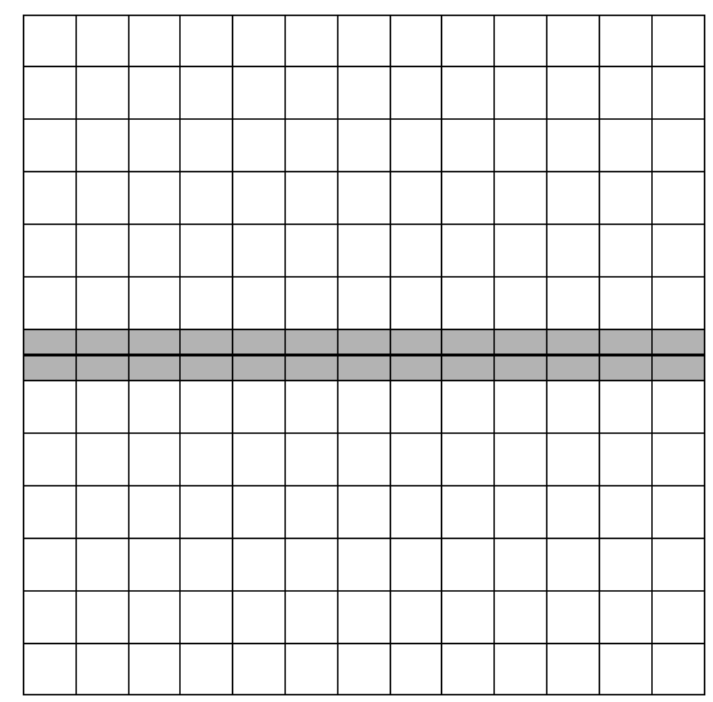

Fig. 7. Viscoelastic half-space discretization. 


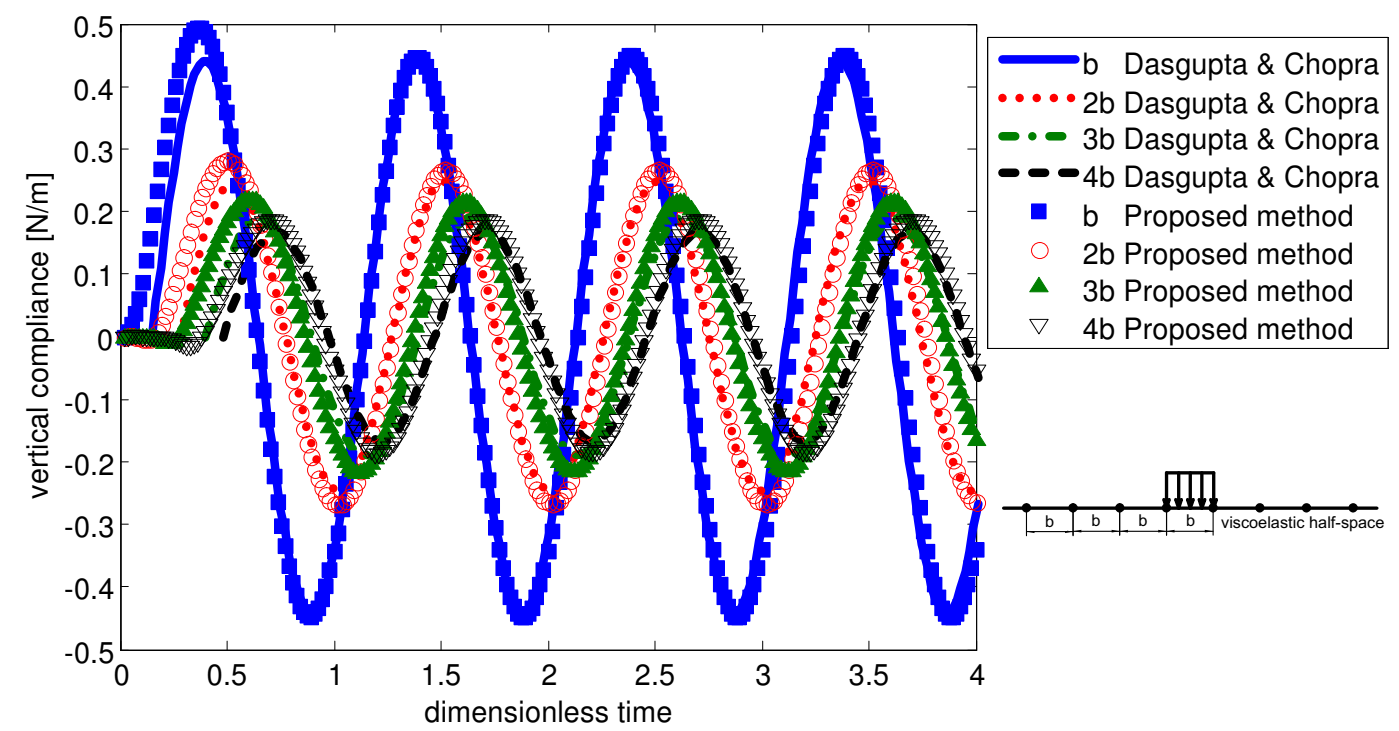

Fig. 8. Vertical compliances of viscoelastic half-space. 


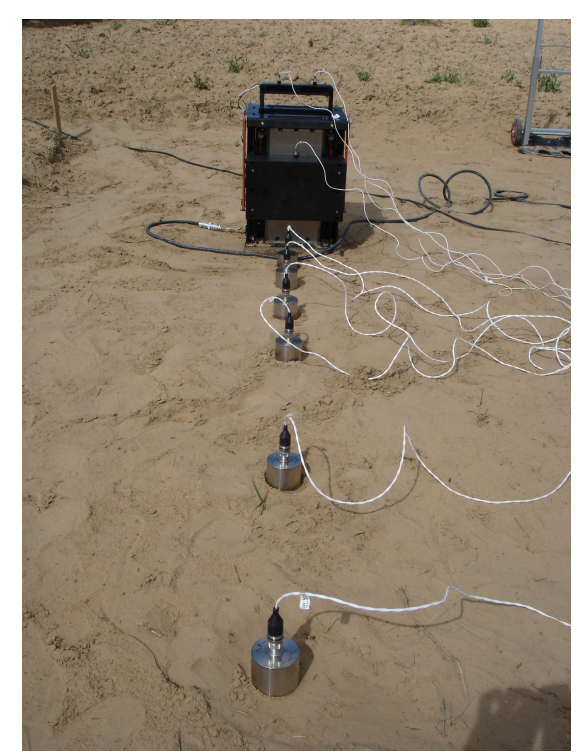

Fig. 9. Experimental setup. 


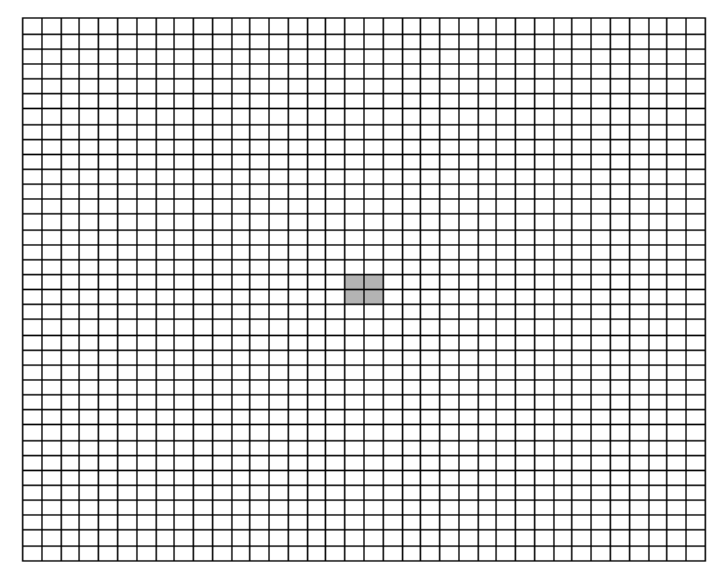

Fig. 10. Soil discretization. 


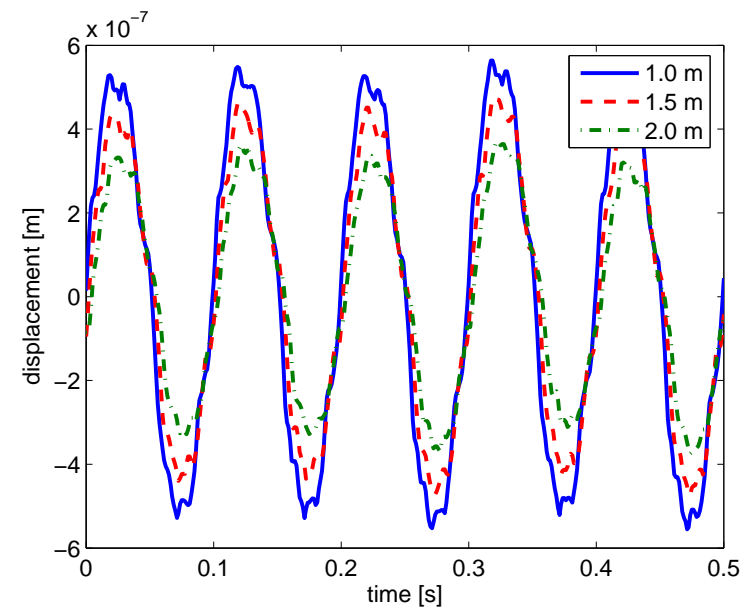

Fig. 11. Experimental vertical displacement. $f=10 \mathrm{~Hz}$. 


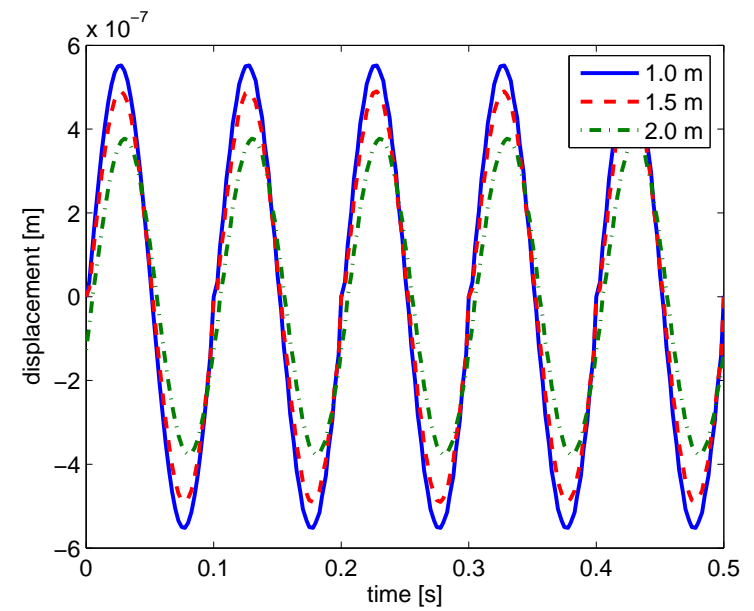

Fig. 12. Numerical vertical displacement. $f=10 \mathrm{~Hz}$. 


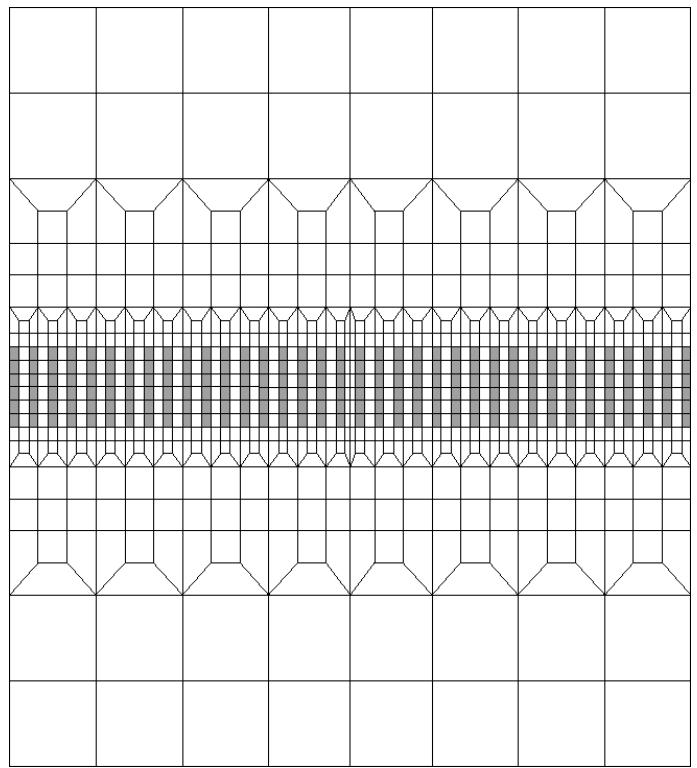

Fig. 13. Discretization of the surface around the track for a length equivalent to 36 sleepers. 


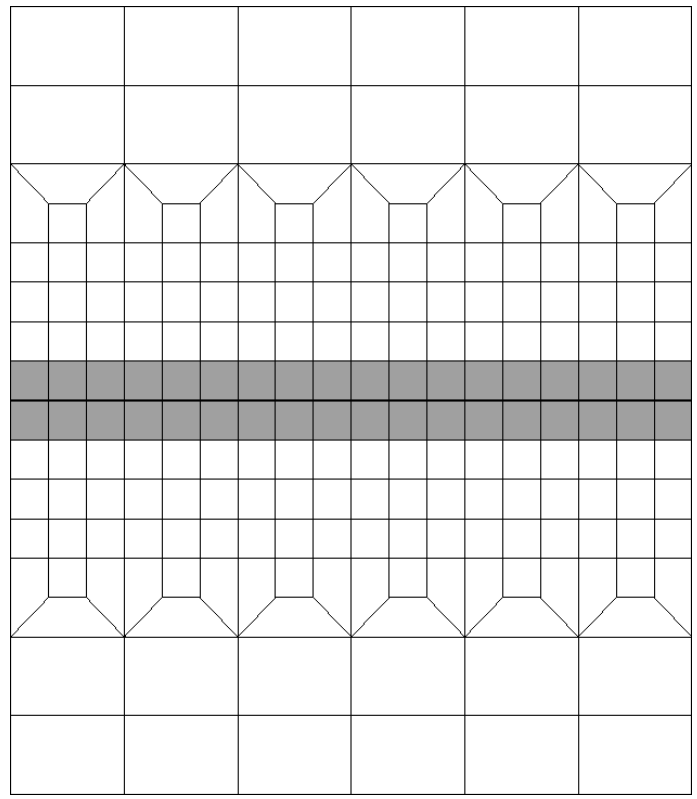

Fig. 14. Boundary element mesh for the proposed model. 


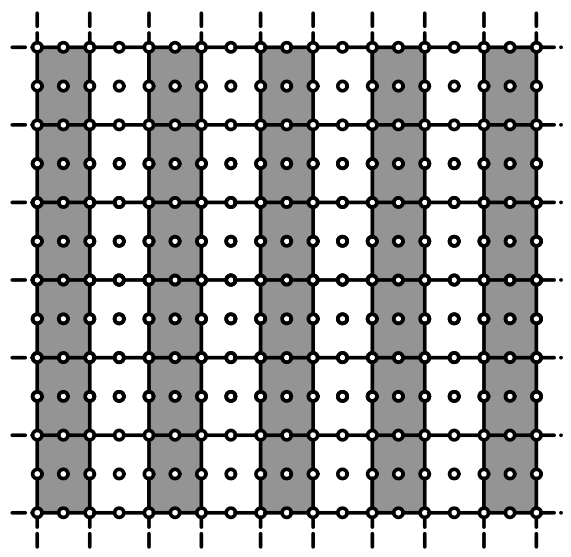

(a) Dense mesh.

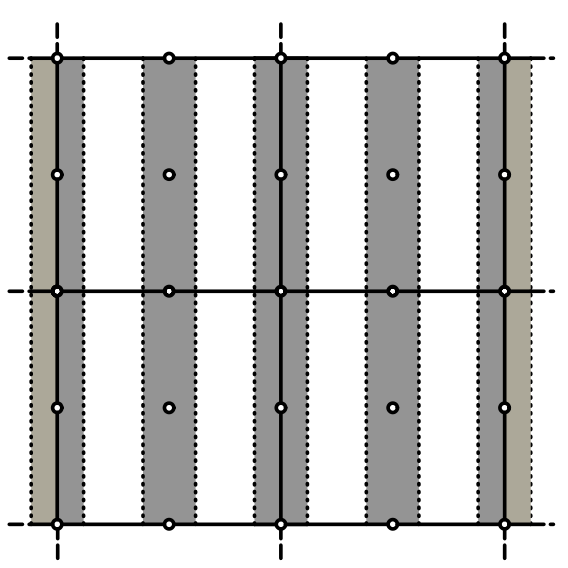

(b) Coarse mesh

Fig. 15. BE discretization of the sleeper zone 


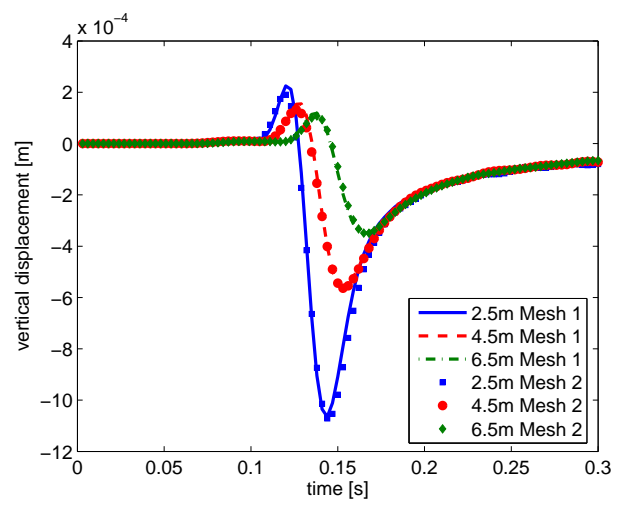

(a) Vertical displacements.

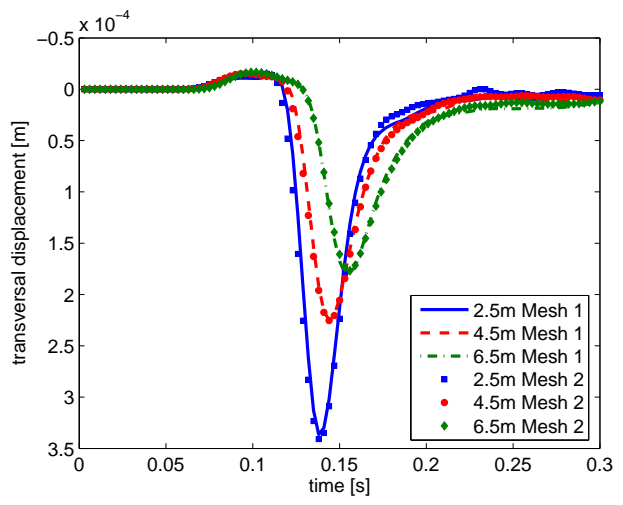

(b) Cross displacements.

Fig. 16. Soil surface displacements: Dense mesh vs. coarse mesh. 


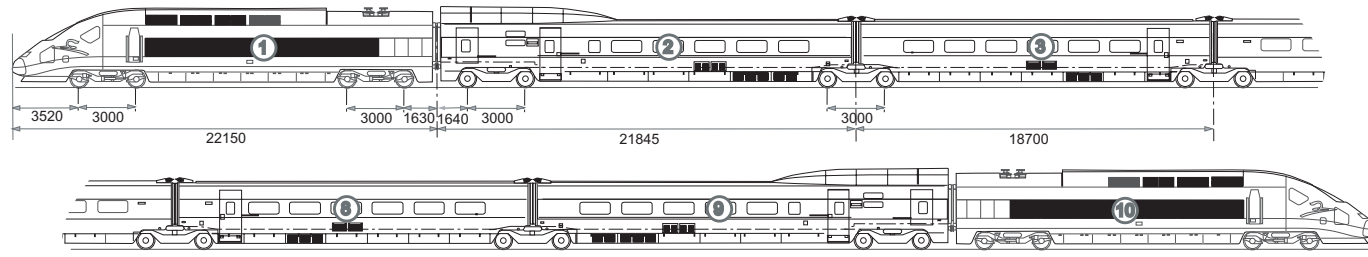

(a)

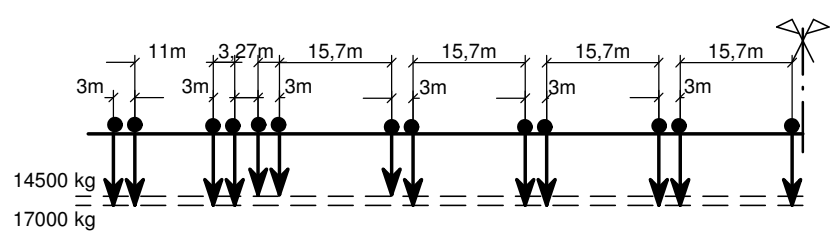

(b)

Fig. 17. Configuration of the Alstom High Speed Train (Thalys-AVE). 


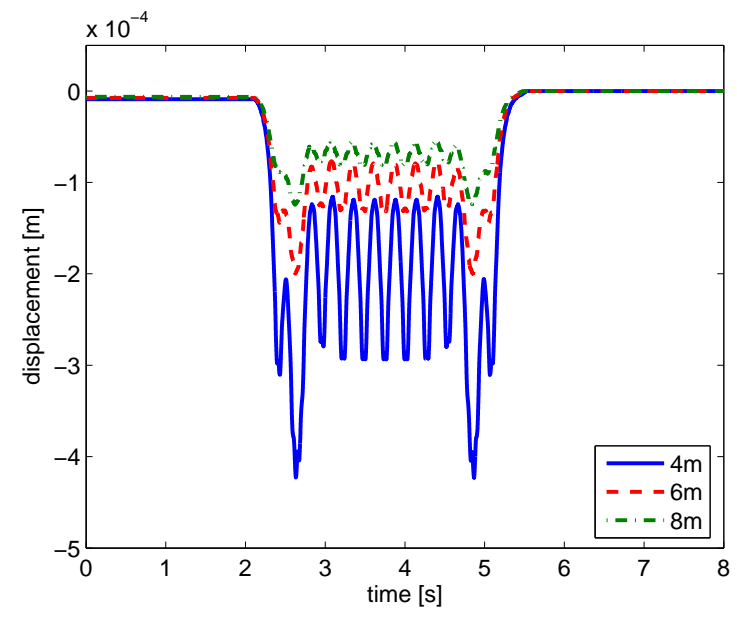

Fig. 18. Soil surface vertical displacements for $v=256 \mathrm{~km} / \mathrm{h}$. 


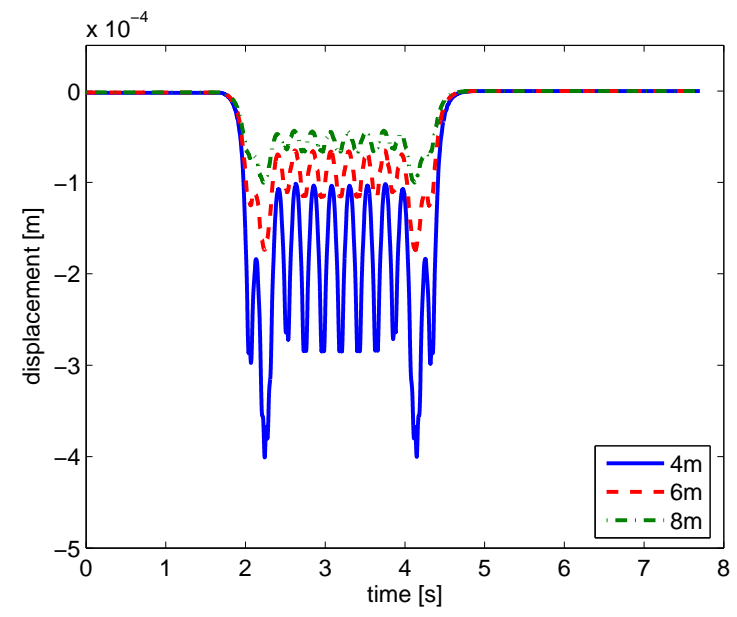

Fig. 19. Soil surface vertical displacements for $v=300 \mathrm{~km} / \mathrm{h}$. 


\section{A Appendix}

The fundamental solution tensors at point $\mathbf{x}$, corresponding to an impulse point load at $\mathbf{x}^{i}$ are [23]:

$$
\begin{aligned}
u_{l k}^{*}\left(\mathbf{x}, t ; \mathbf{x}^{i}\right) & =\frac{1}{4 \pi \rho}\left\{\frac{t}{r^{2}}\left(\frac{3 r_{, l} r_{, k}}{r}-\frac{\delta_{l k}}{r}\right)\left[H\left(t-\frac{r}{c_{p}}\right)-H\left(t-\frac{r}{c_{s}}\right)\right]\right\} \\
& +\frac{1}{4 \pi \rho}\left\{\frac{r_{, l} r_{, k}}{r}\left[\frac{1}{c_{p}^{2}} \delta\left(t-\frac{r}{c_{p}}\right)-\frac{1}{c_{s}^{2}} \delta\left(t-\frac{r}{c_{s}}\right)\right]+\frac{\delta_{l k}}{r c_{s}^{2}} \delta\left(t-\frac{r}{c_{s}}\right)\right\}
\end{aligned}
$$

where $r=\left|\mathbf{x}-\mathbf{x}^{i}\right|$, and

$$
p_{l k}^{*}\left(\mathbf{x}, t ; \mathbf{x}^{i}\right)=\frac{1}{4 \pi}\left\{\left(\frac{\partial r}{\partial n} \delta_{l k}+r_{, k} n_{l}\right) A+r_{, l} r_{, k} \frac{\partial r}{\partial n} B+r_{, l} n_{k} C\right\}
$$

where,

$$
\begin{aligned}
A & =\frac{6 c_{s}^{2}}{r^{4}} t\left[H\left(t-\frac{r}{c_{p}}\right)-H\left(t-\frac{r}{c_{s}}\right)\right]-\frac{2}{r^{2}}\left[\delta\left(t-\frac{r}{c_{s}}\right)-\left(\frac{c_{s}}{c_{p}}\right)^{2} \delta\left(t-\frac{r}{c_{p}}\right)\right] \\
& -\frac{1}{r^{2}}\left[\delta\left(t-\frac{r}{c_{s}}\right)-\frac{r}{c_{s}} \dot{\delta}\left(t-\frac{r}{c_{s}}\right)\right] \\
B & =\frac{-30 c_{s}^{2}}{r^{4}} t\left[H\left(t-\frac{r}{c_{p}}\right)-H\left(t-\frac{r}{c_{s}}\right)\right]+\frac{12}{r^{2}}\left[\delta\left(t-\frac{r}{c_{s}}\right)-\left(\frac{c_{s}}{c_{p}}\right)^{2} \delta\left(t-\frac{r}{c_{p}}\right)\right] \\
& +\frac{2}{r c_{s}}\left[\dot{\delta}\left(t-\frac{r}{c_{s}}\right)-\left(\frac{c_{s}}{c_{p}}\right)^{3} \dot{\delta}\left(t-\frac{r}{c_{p}}\right)\right] \\
C & =\frac{6 c_{s}^{2}}{r^{4}} t\left[H\left(t-\frac{r}{c_{p}}\right)-H\left(t-\frac{r}{c_{s}}\right)\right]-\frac{2}{r^{2}}\left[\delta\left(t-\frac{r}{c_{s}}\right)-\left(\frac{c_{s}}{c_{p}}\right)^{2} \delta\left(t-\frac{r}{c_{p}}\right)\right] \\
& -\frac{1}{r^{2}}\left[1-2\left(\frac{c_{s}}{c_{p}}\right)^{2}\right]\left[\delta\left(t-\frac{r}{c_{p}}\right)+\frac{r}{c_{p}} \dot{\delta}\left(t-\frac{r}{c_{p}}\right)\right]
\end{aligned}
$$

